\title{
Numerical Simulation Of Supersonic Compression Ramp Flow
}

\author{
Ki-Hyuk Yang ${ }^{1}$, Jong-Dae Lee ${ }^{1}$, Seung-Won Lee ${ }^{1}$, Hak-Yoon Kim ${ }^{1}$, Dong-Jin Sheen ${ }^{1}$, Chang-Hwan Park ${ }^{* 1}$ \\ ${ }^{1}$ Aeronautical System Engineering, Hanseo University, Taean, Chungcheongnam-do, 32158, Republic of Korea \\ *Corresponding author, E-mail: lars@hanseo.ac.kr
}

Article History:Received:11 november 2020; Accepted: 27 December 2020; Published online: 05 April 2021

ABSTRACT : Shock wave/boundary layer interaction (SWBLI) is a highly critical problem that occurs in aircraft in transonic or supersonic flow. This study performed CFD analysis of the supersonic ramp flow of freestream Mach number 2.79. To secure reliability of the CFD analysis, adaptive mesh refinement using a gradient $p$ sensor was used. Through this, a grid of sufficient resolution was obtained for the region of shock wave, expansion wave, and flow separation. The prediction performance of 7 turbulence models that are widely used in engineering application were compared. The baseline k- $\omega$ twoequation model showed the best prediction performance, while the SST k- $\omega$ model, which is one of the most widely used two-equation models, and the 2 Reynolds stress models showed relatively poor prediction performance. In the SWBLI problem, the use of adaptive mesh refinement made it possible to secure sufficient grid resolution; meanwhile, comparison of the prediction performance of the various turbulence models confirmed that for the SWBLI problem, the generally used turbulence model was somewhat inappropriate.

Keywords:supersonic compression ramp flow; computational fluid dynamics (CFD); compressible turbulence; turbulence model; adaptive mesh refinement; shock wave/boundary layer interaction; FLUENT

\section{Introduction}

Shock wave/boundary layer interaction (SWBLI) occurs when a shock wave (oblique shock or normal shock) penetrates the boundary layer in transonic or supersonic flow, and is observed in various shapes, such as the outer shape of the aircraft, the engine inlet, and the fins of rockets. Supersonic ramp flow is a representative problem of SWBLI, and adverse pressure gradients are formed by the ramp shape, resulting in separation of the boundary layer. This again directly causes mutual interference with the shock wave; and here, accurate predictions of the pressure distribution, friction coefficient, and heat transfer coefficient on the wall are very important in engineering application.

In this study, using ANSYS FLUENT, one of the most widely used commercial CFD software, we analyzed compression ramp flow, a representative problem of SWBLI, and analyzed the predictive capability of various turbulence models available in FLUENT.

\section{Materials and Methods}

Many experimental studies have been conducted on ramp flows of various angles[1,2,3,4], and this study covered only the $20^{\circ}$ inclination shape with sufficient flow separation[5]. Figure 1 shows a schematic of the flow, while

Table 1 shows freestream conditions that include freestream Mach number=2.79.

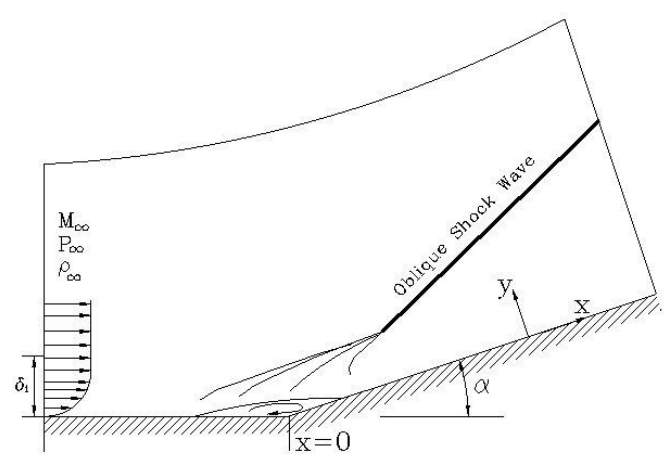

Figure 1. Schematic of the supersonic compression ramp flow.

Table 1. Freestream condition.

\begin{tabular}{ll}
$R e_{\infty} / m$ & $6.3 \times 10^{7} / m$ \\
\hline
\end{tabular}

*Corresponding author: Chang-Hwan Park

Aeronautical System Engineering, Hanseo University, Taean, Chungcheongnam-do, 32158, Republic of Korea E-mail: lars@hanseo.ac.kr 


\begin{tabular}{cc}
\hline$M_{\infty}$ & 2.79 \\
$U_{\infty}$ & $562 \mathrm{~m} / \mathrm{s}$ \\
$P_{0}$ & $6.9 \times$ \\
$T_{0}$ & $10^{5} \mathrm{~Pa}$ \\
\hline
\end{tabular}

\subsection{Governing equation \& turbulence model}

ANSYS FLUENT 2019 R3 was used to obtain the solution of the two-dimensional compressible NavierStokes equation. To obtain the solution of steady compressible flow, FLUENT's density-based solver was used, and the AUSM+ scheme, which shows excellent performance in shock wave calculation, was selected for the inviscid flux. Pressure was calculated using the ideal gas state equation from the solution of the Navier-Stokes equation, and viscosity was determined from the empirical Sutherland's formula.

Table 2 shows the 7 models that are widely used in engineering application that were selected, among the turbulence models built into FLUENT[6]. The Spalart-Allmaras (SA) one-equation model[7], a realizable k- $\varepsilon$ model (high-Reynolds number model)[8] that uses a wall function, 3 types of $\mathrm{k}-\omega$ models that are low Reynolds turbulence models[9,10], and 2 Reynolds-stress models[6,9] were selected.

Table 2. Turbulence models.

\begin{tabular}{c|c} 
Turbulence model & Type \\
\hline Spalart-Allmaras (S-A) model & One-equation model \\
\hline Realizable $k-\varepsilon$ model & $\begin{array}{c}\text { High Reynolds number } \\
\text { two-equation model }\end{array}$ \\
\hline Standard $k-\omega$ model & $\begin{array}{c}\text { Low Reynolds number } \\
\text { two-equation model }\end{array}$ \\
\hline Baseline (BSL) $k-\omega$ model & $\begin{array}{c}\text { Reynolds stress model } \\
\text { Shear-Stress-Transport (SST) } k-\omega \text { model }\end{array}$ \\
\hline Stress-omega model & \\
\hline Stress-BSL model & \\
\hline
\end{tabular}

\subsection{Computational Domain and Boundary condition}

A quadrilateral grid was used for the grid, and the size of the computational domain was expressed as dimensionless as inlet boundary layer thickness $\delta_{i}$ (see Figure 1). The velocity inlet boundary condition (BC) was used on the left side, while the pressure outlet condition was used on the right side. In the case of supersonic flow, the pressure outlet condition of FLUENT is processed by extrapolating flow variables from values inside the calculation area. The upper side used a pressure far-field condition, which is a characteristic boundary condition; and for this purpose, the size of the calculation area in the y direction was set sufficiently large at $10 \delta_{i}$.

Table 3 shows the conditions for various flow variables that are presented at the $\mathrm{x}=-2 \delta_{i}$ position in the experiment[4,5], which conditions can be used as an inlet condition for CFD analysis. In this study, ramp flow analysis was performed using various turbulence models, and certain turbulence models sometimes predict the separation bubble excessively larger than the actual one[11]. To overcome this problem, in this study, the calculation area on the left inlet side was extended from $-2 \delta_{i}$ to $-4 \delta_{i}$. The inlet condition was obtained by solving the compressible boundary layer equation, and EDDYBL[9] software was used for this purpose. In the calculation of EDDYBL, the position where the momentum Reynolds thickness number $\operatorname{Re}_{\theta}$ of

Table 3 satisfies 78,000 was found, and the upstream condition of $2 \delta_{i}$ from this position was used as the inlet condition at the $-4 \delta_{i}$ position. 


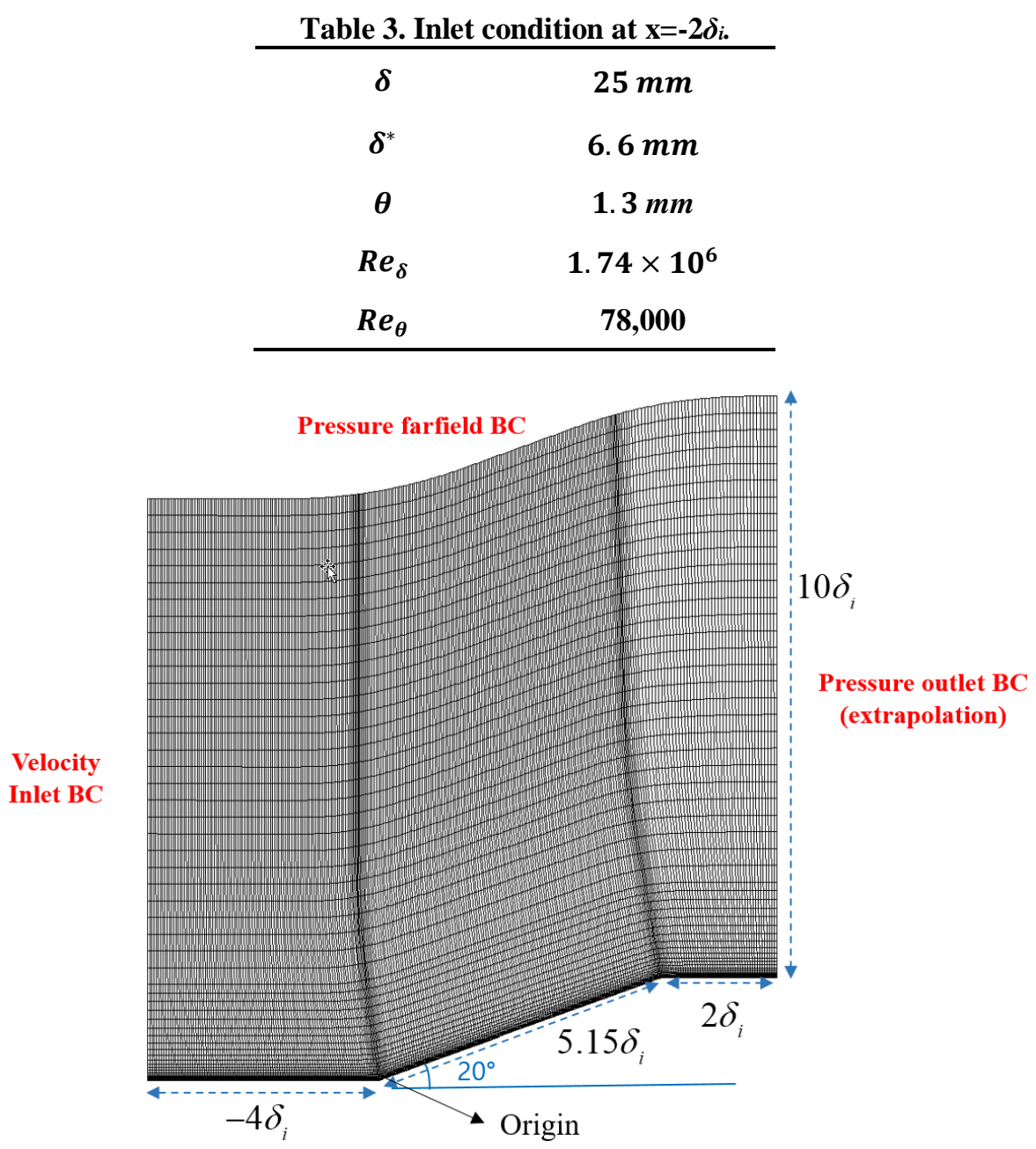

Figure 2. Computational grid \& boundary condition.

\subsection{Grid independence test}

In computational fluid dynamics (CFD) simulation, the grid independence test is a very important process to secure the repeatability/reproducibility of the analysis, and obtain reliability of calculation[12]. In this study, $282 \times 70$ grids were selected as the base mesh through preliminary study, and Figure 2 shows the base mesh. The grid test was performed using the SST $\mathrm{k}-\omega$ turbulence model[10].

The total number of grids was maintained at the same number as the base mesh, and the grid was created so that only the $y+$ value was changed on the wall, and the results were compared. For the 4 grids in Table 4, the number of grids is the same, but the maximum value of $y^{+}$is different at the wall. $y_{\text {max }}^{+}$is an approximate representation of the largest value among $y_{\max }^{+}$values along the wall. From the analysis results, the lift coefficient $C_{L}$ and drag coefficient $C_{D}$ on the wall are shown. In Case 1, the y-direction grid is clustered the most toward the wall, so the $y^{+}$value is the lowest. Table 4 shows the results of $C_{L}$ and $C_{D}$, as well as the error based on case 1 . The errors of $C_{L}$ and $C_{D}$ are not very large, with a maximum of $(0.11$ and 0.46$) \%$, respectively.

Table 4. Grid test 1

\begin{tabular}{ccccccc}
\hline Case name & No. of cells & $\mathrm{y}^{+}$max & $\mathrm{C}_{\mathrm{L}}$ & Error of $\mathrm{C}_{\mathrm{L}}$ & $\mathrm{C}_{\mathrm{D}}$ & Error of $\mathrm{C}_{\mathrm{D}}$ \\
\hline case 0 (Base) & $279 \times 68$ & 0.7 & $-5.312 \mathrm{E}-02$ & $0.08 \%$ & $1.614 \mathrm{E}-02$ & $0.33 \%$ \\
case 1 & $279 \times 68$ & 0.35 & $-5.307 \mathrm{E}-02$ & - & $1.609 \mathrm{E}-02$ & - \\
case 2 & $279 \times 68$ & 4 & $-5.313 \mathrm{E}-02$ & $0.11 \%$ & $1.616 \mathrm{E}-02$ & $0.45 \%$ \\
case 3 & $279 \times 68$ & 40 & $-5.303 \mathrm{E}-02$ & $-0.08 \%$ & $1.602 \mathrm{E}-02$ & $-0.46 \%$ \\
\hline
\end{tabular}

Figure 3 and Figure 4 show the wall skin friction and wall pressure distribution calculated by each grid. 
The SST k- $\omega$ model used in the grid test predicts the separation bubble size larger than the experiment. Except for case 3 where $\mathrm{y}_{\max }^{+}=40$, it is evident that both the skin friction coefficient and the wall pressure distribution show almost the same results. In general, it is known that for the low Reynolds number turbulence model, it is recommended that the $\mathrm{y}^{+}$value be less than 1 [12]. In fact, since the $\mathrm{y}_{\max }^{+}$value shown in Table 4 is the maximum value of $\mathrm{y}^{+}$along all the walls, the $\mathrm{y}+$ value of most wall regions in case 2 , where $\mathrm{y}_{\max }^{+}=4$, is less than 1 .

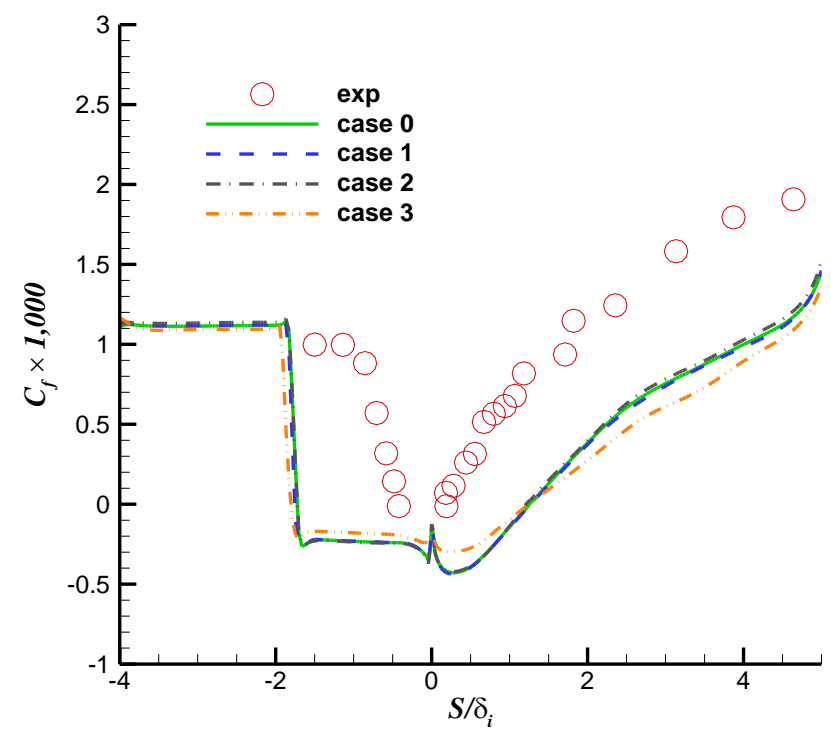

Figure 3. Skin friction coefficient distribution.

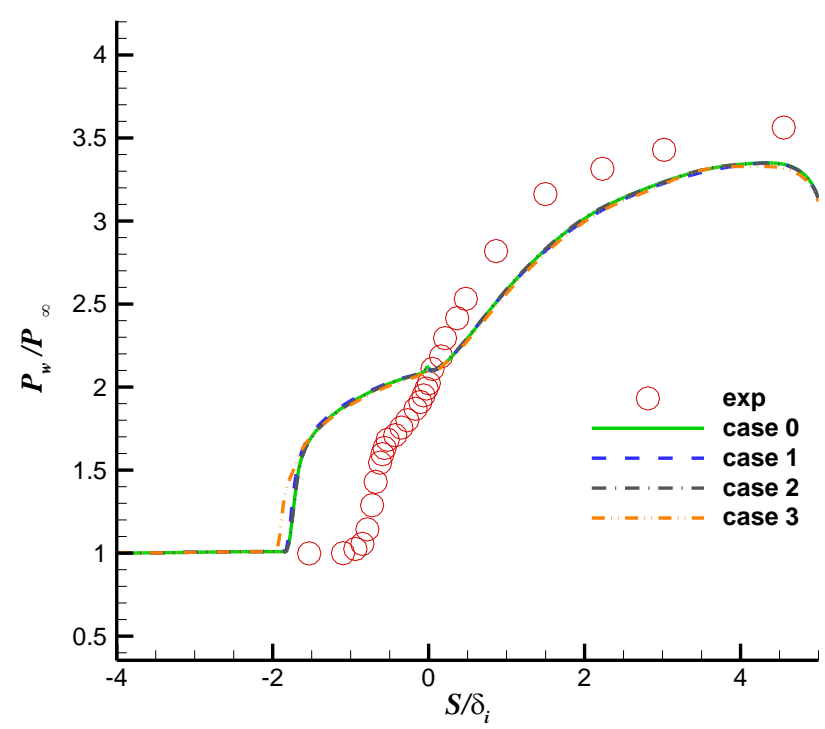

Figure 4. Wall pressure distribution.

The above results confirmed that the $y^{+}$value of case 0 , the base mesh, is sufficient for the analysis of the ramp flow covered in this study; all $y^{+}$values were fixed to the same level as the base mesh (case 0); and the total number of grids was changed, and a grid test was performed. Table 5 shows the case of changing the number of grids and the lift coefficient $C_{L}$ and drag coefficient $C_{D}$ obtained from the analysis results. The errors of $C_{L}$ and $C_{D}$ calculated based on the A6 case with the largest number of grids show that there is no significant difference overall, with maximum errors of $(0.14$ and 0.63$) \%$, respectively.

Table 5. Grid test 2

\begin{tabular}{ccccccc}
\hline Case name & No. of grids & $y^{+}{ }_{\max }$ & $\mathrm{C}_{\mathrm{L}}$ & Error of $\mathrm{C}_{\mathrm{L}}$ & $\mathrm{C}_{\mathrm{D}}$ & Error of $\mathrm{C}_{\mathrm{D}}$ \\
\hline A1 & $140 \times 48$ & 0.7 & $-5.321 \mathrm{E}-02$ & $0.14 \%$ & $1.628 \mathrm{E}-02$ & $0.63 \%$ \\
A2 & $249 \times 48$ & 0.7 & $-5.314 \mathrm{E}-02$ & $0.01 \%$ & $1.622 \mathrm{E}-02$ & $0.24 \%$
\end{tabular}




\begin{tabular}{ccccccc} 
case 0 (Base) & $279 \times 68$ & 0.7 & $-5.312 \mathrm{E}-02$ & $-0.04 \%$ & $1.614 \mathrm{E}-02$ & $-0.22 \%$ \\
A3 & $299 \times 88$ & 0.7 & $-5.316 \mathrm{E}-02$ & $0.04 \%$ & $1.615 \mathrm{E}-02$ & $-0.18 \%$ \\
A4 & $319 \times 108$ & 0.7 & $-5.311 \mathrm{E}-02$ & $-0.05 \%$ & $1.616 \mathrm{E}-02$ & $-0.09 \%$ \\
A5 & $339 \times 128$ & 0.7 & $-5.315 \mathrm{E}-02$ & $0.02 \%$ & $1.619 \mathrm{E}-02$ & $0.10 \%$ \\
A6 & $359 \times 148$ & 0.7 & $-5.314 \mathrm{E}-02$ & - & $1.618 \mathrm{E}-02$ & - \\
\hline
\end{tabular}

Figure 5 and Figure 6 compare the skin friction coefficient and wall pressure distribution, respectively. Except for the coarsest mesh, A1, the results are almost the same. Thus, it was confirmed that case 0 , which was selected as the base mesh in this study, is an appropriate grid that provides sufficient resolution for the analysis of ramp flow.

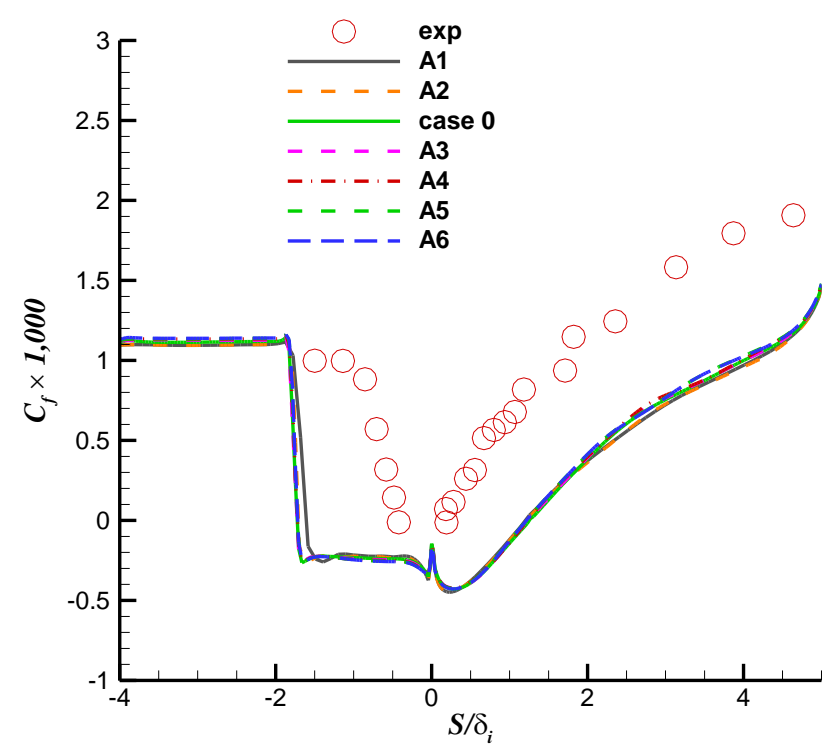

Figure 5. Skin friction coefficient distribution.

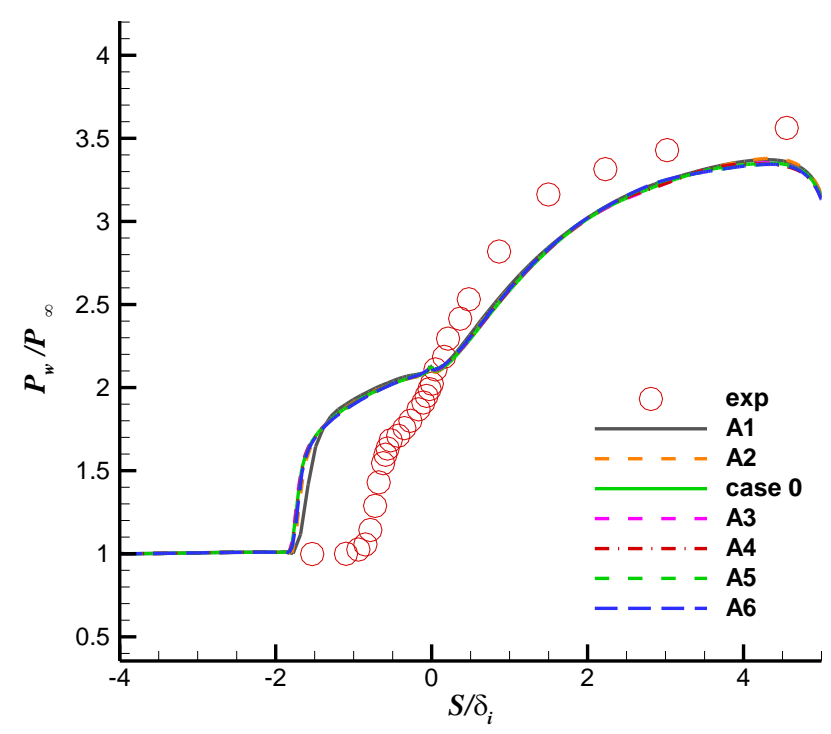

Figure 6. Wall pressure distribution.

\section{Results and Discussion}




\subsection{Adaptive mesh refinement}

The grid test in Section 2.3 is a very important process to secure the reliability of analysis in CFD analysis. Figure 7 compares the static pressure contours of three cases among the cases in Table 5. The number of grids increases in the order: (a) A1 < (b) base mesh < (c) A6; if the number of grids is small, it can be confirmed that the resolution of the grid near the shock wave or expansion wave is insufficient; and in the case of A6 with the largest number of grids, the discontinuity of the shock wave is best resolved. As already mentioned in Section 2.3, all of the cases in Table 5 have already been selected so that the $y^{+}$value near the wall is less than 1 , and the grid resolution in the $x$ direction is sufficiently secured. Thus, it can be seen that even the grid selected through the grid independence test may not have sufficient resolution near the shock wave.

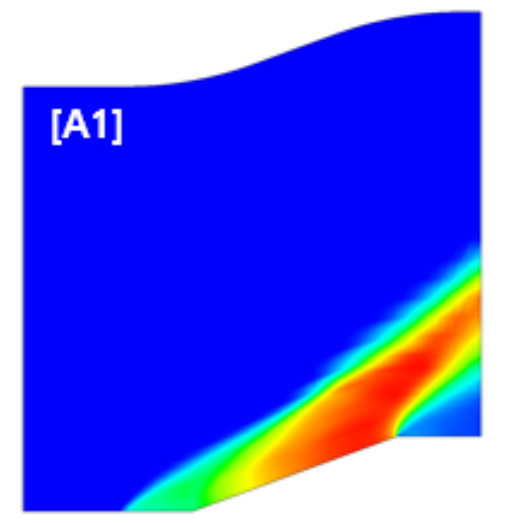

(a) A1: $141 \times 50$

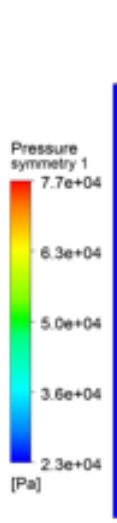

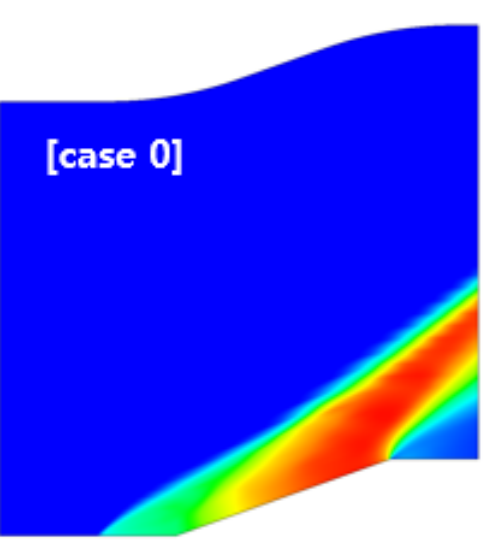

(b) Case 0: $282 \times 70$

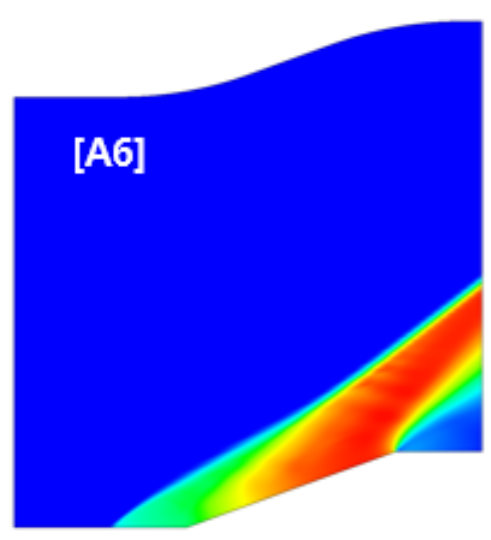

(c) A6: $360 \times 150$

Figure 7. Comparison of static pressure contour.

In the case of having local discontinuity characteristics like a shock wave, the problem arises that when the method of global refinement is used, the number of final grids is excessively large, as in Section 2.3. Therefore, it is necessary to selectively concentrate the grid in the local region where the shock wave is generated; and in this study, an adaptive mesh refinement method using a gradient $\mathrm{p}$ sensor was used [13,14]. By using a gradient $\mathrm{p}$ sensor to find an area with a large change in static pressure, and increasing the grid of that area after the first adaptive mesh refinement (level 1), CFD analysis was performed; and mesh refinement (level 2) was performed, up to level 3 .

Table 6 shows the cell number and $C_{L}$ and $C_{D}$ values of the grid for each level. As mentioned in Section 2.3, the base mesh has already sufficiently resolved the flow near the wall, so it has little effect on the $C_{L}$ and $C_{D}$ obtained by integrating the pressure and shear stress near the wall. With the same concept, looking at the skin friction coefficient and wall pressure distribution in Figure 8, it is evident that the adaptive mesh refinement hardly affects the flow characteristics near the wall.

Table 6. Adaptive mesh refinement with $\nabla p$ sensor.

\begin{tabular}{cccccc}
\hline Case name & No. of cells & $C_{L}$ & Error of $C_{L}$ & $C_{D}$ & Error of $C_{D}$ \\
\hline case 0 (Base) & 18,972 & $-5.312 \mathrm{E}-02$ & $0.11 \%$ & $1.6143 \mathrm{E}-02$ & $-0.02 \%$ \\
level 1 & 23,247 & $-5.311 \mathrm{E}-02$ & $0.10 \%$ & $1.6169 \mathrm{E}-02$ & $0.14 \%$ \\
level 2 & 32,673 & $-5.306 \mathrm{E}-02$ & $-0.01 \%$ & $1.6149 \mathrm{E}-02$ & $0.02 \%$ \\
level 3 & 55,830 & $-5.306 \mathrm{E}-02$ & - & $1.6146 \mathrm{E}-02$ & - \\
\hline
\end{tabular}




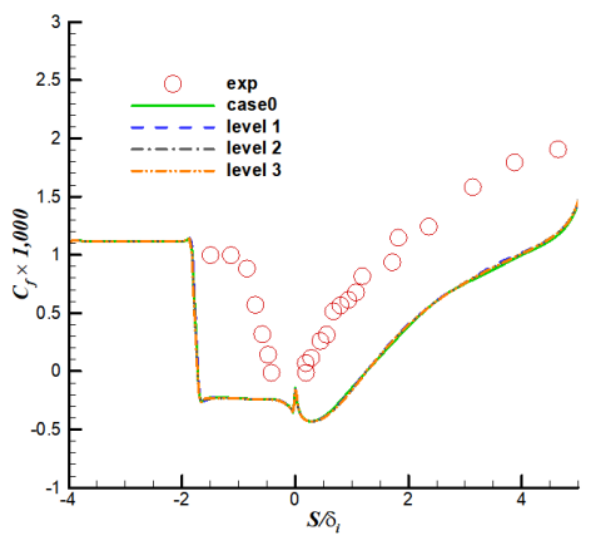

(a) Skin friction coefficient

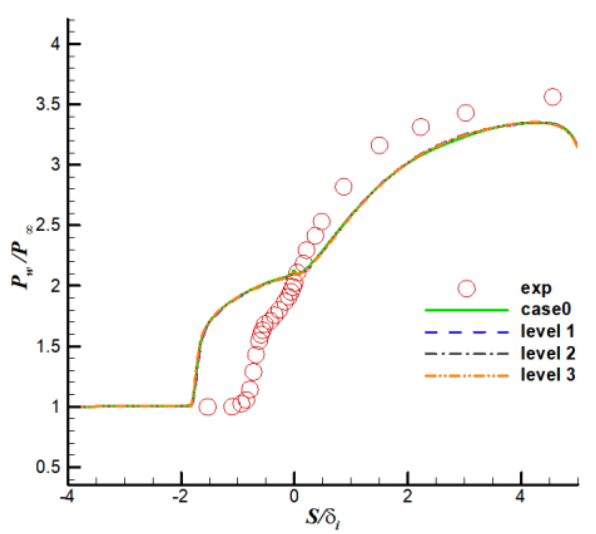

(b) wall pressure

Figure 8. Skin friction coefficient \& wall pressure.

Figure 9 shows the grid and pressure contour for each level. As the level of mesh refinement increases, a larger number of grids are created in the region where the pressure gradient value is large. It can also be seen that the gradient $\mathrm{p}$ sufficiently captures the positions of shock waves and expansion waves undergoing rapid changes in pressure, and refines the grid of those parts.

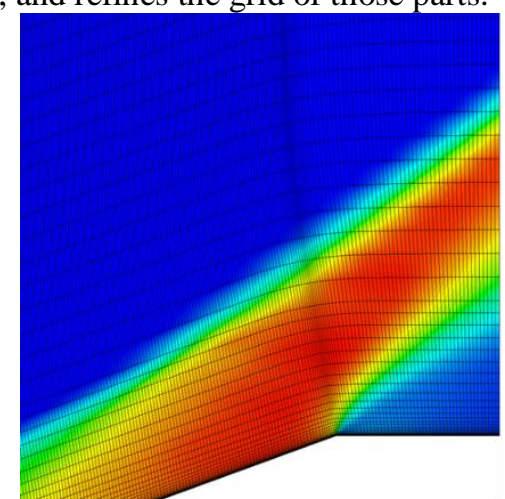

(a) Case 0: 18,927 cells

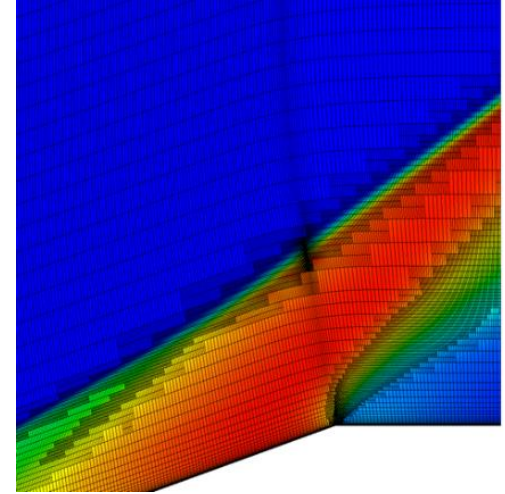

(c) level 2: 32,673 cells

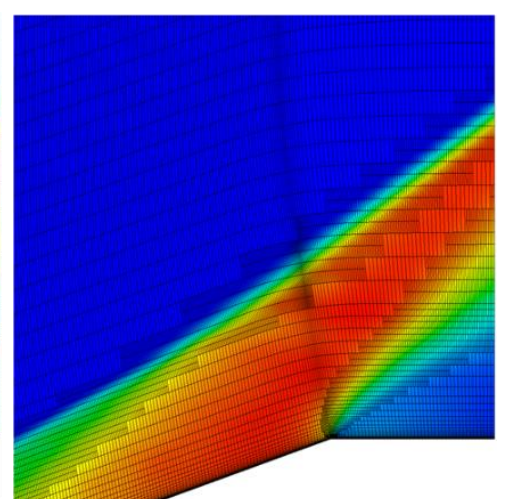

(b) level $1: 23,247$ cells

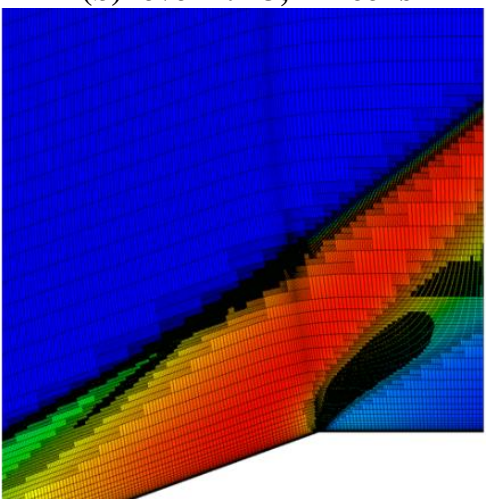

(d) level 3: 55,830 cells

Figure 9. Adaptive mesh refinement (static pressure contour). 


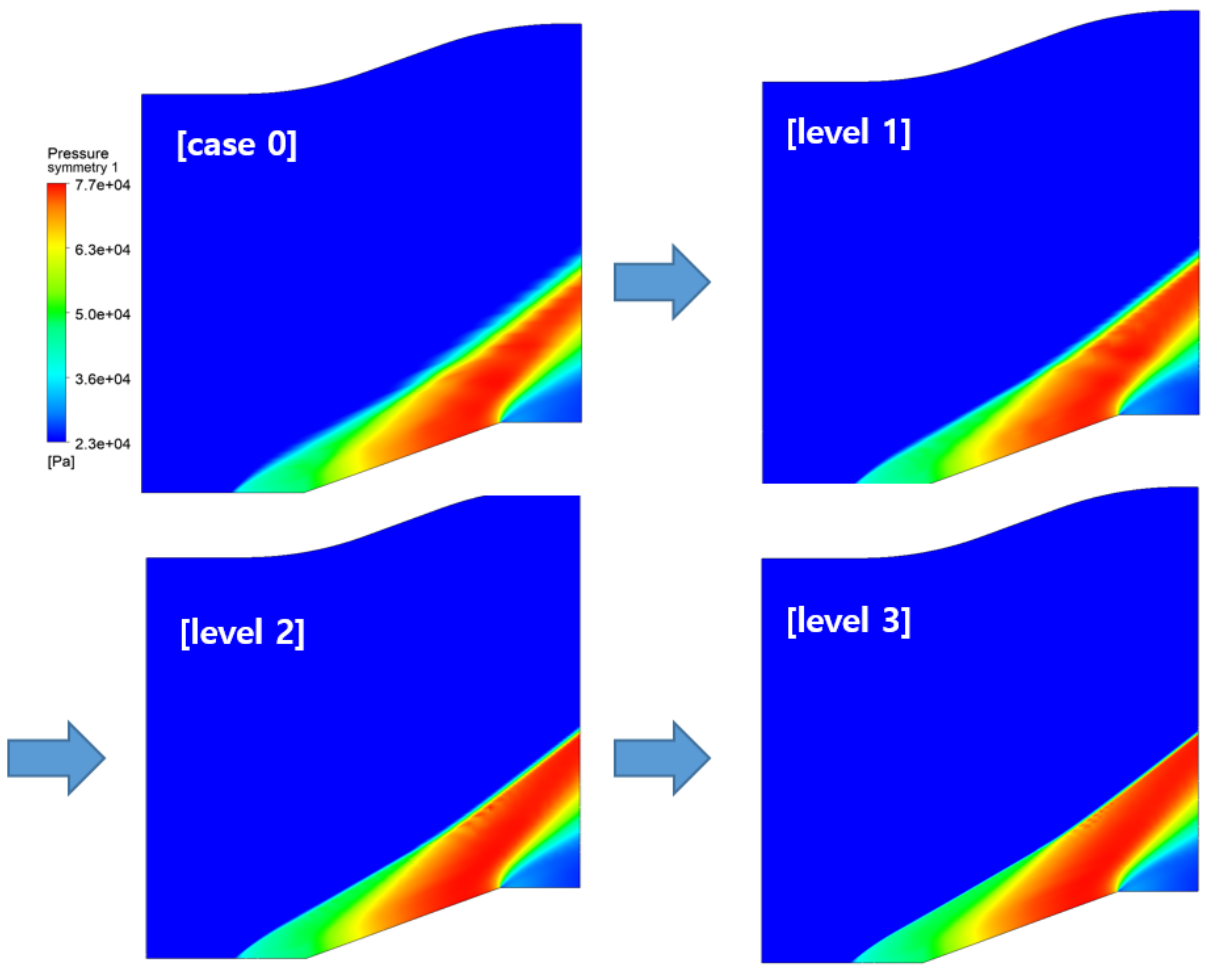

Figure 10. Variation of pressure contour according to the mesh refinement level.

Figure 10 shows the change of the pressure contour plot according to the refinement level of the grid; it can be seen that as the level increases, the thickness of the shock wave decreases, and phenomena, such as the oscillation pattern of the flow solution before and after the shock wave, decrease. Figure 11(a) indicates the position of line A passing through the shock wave, while Figure 11(b) shows the change in pressure along line A. As the mesh refinement level increases, discontinuous and abrupt changes of the pressure before and after the shock wave can be confirmed. Considering that the total number of cells in Level 3 is 55,830, and that the total number of cells in the A6 case in Table 5 is of a similar level at 53,132, it can be seen that adaptive mesh refinement is a very effective method for securing the appropriate grid resolution. Thus, it can be seen that adaptive mesh refinement capable of local refinement is very useful for shock wave analysis, rather than the grid independence test performing general global refinement.

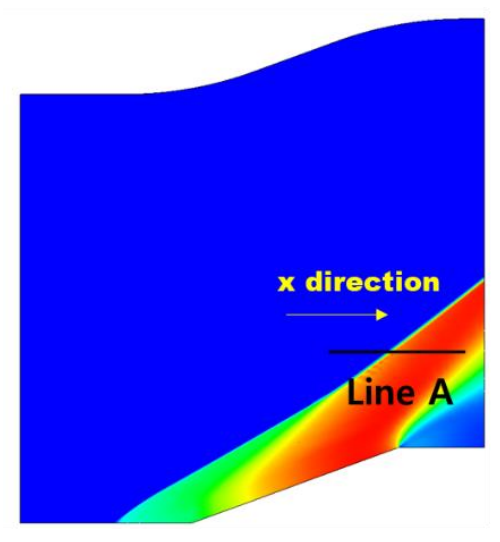

(a) Location of line A

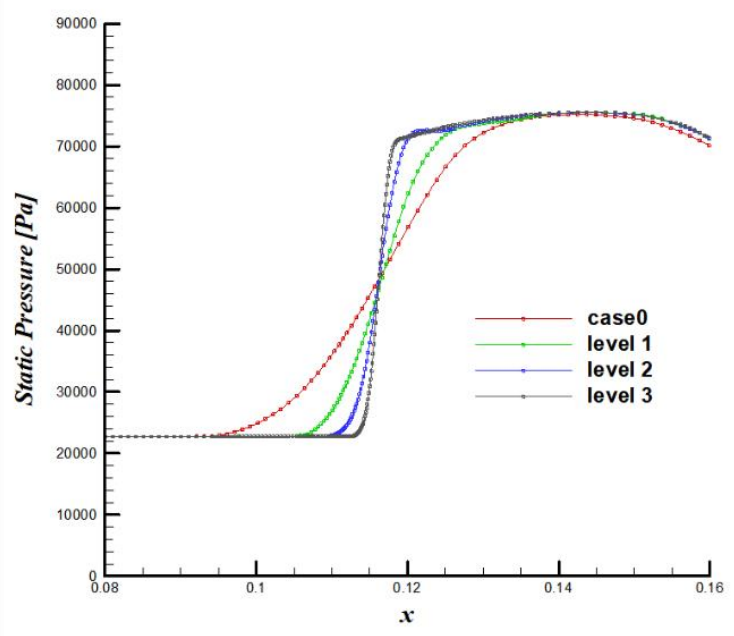

(b) Pressure variation at line A

Figure 11. Pressure variation at line A.

\subsection{Evaluation of various turbulence models}

The ramp flow was analyzed using the various turbulence models shown in 
Table 2. For the analysis of each turbulence model, individual inlet conditions were created using EDDYBL. Figure 12 and Figure 13 show the prediction wall pressure and skin friction coefficient results, respectively, of the S-A one-equation turbulence model and the 3 low Reynolds number turbulence models. For both the wall pressure and skin friction coefficient, the BSL k- $\omega$ model shows the most similar results to the experimental values. Next, the standard $\mathrm{k}-\omega$ model shows excellent results, which predict the flow separation region slightly larger than the experiment. The S-A model not only predicts the size of the separation bubble to be larger than the experimental value, but also predicts the skin friction coefficient after reattachment to be excessively low. The SST k- $\omega$ model predicts the largest separation bubble size and the very low skin friction coefficient after reattachment; and among the four models, the prediction performance is the poorest.

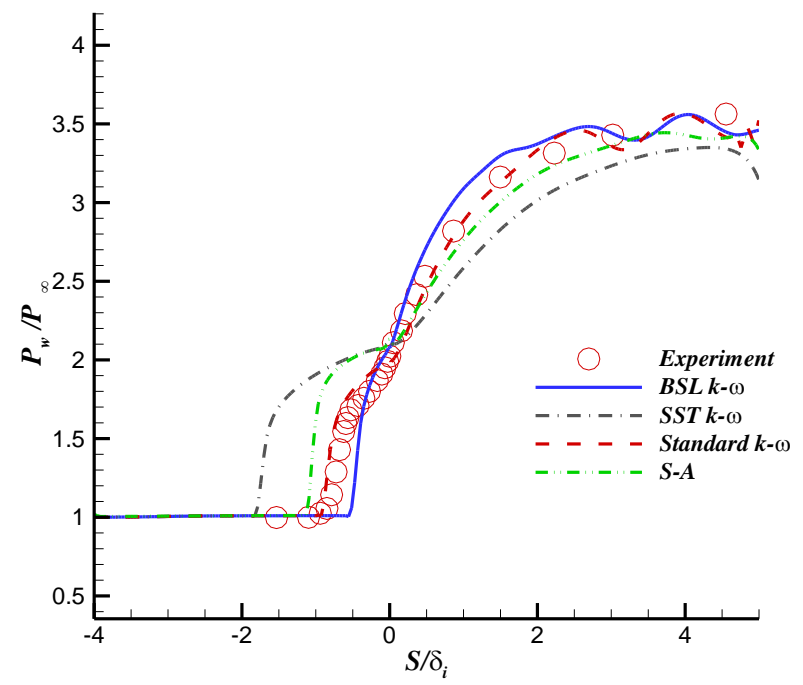

Figure 12. Wall pressure distribution.

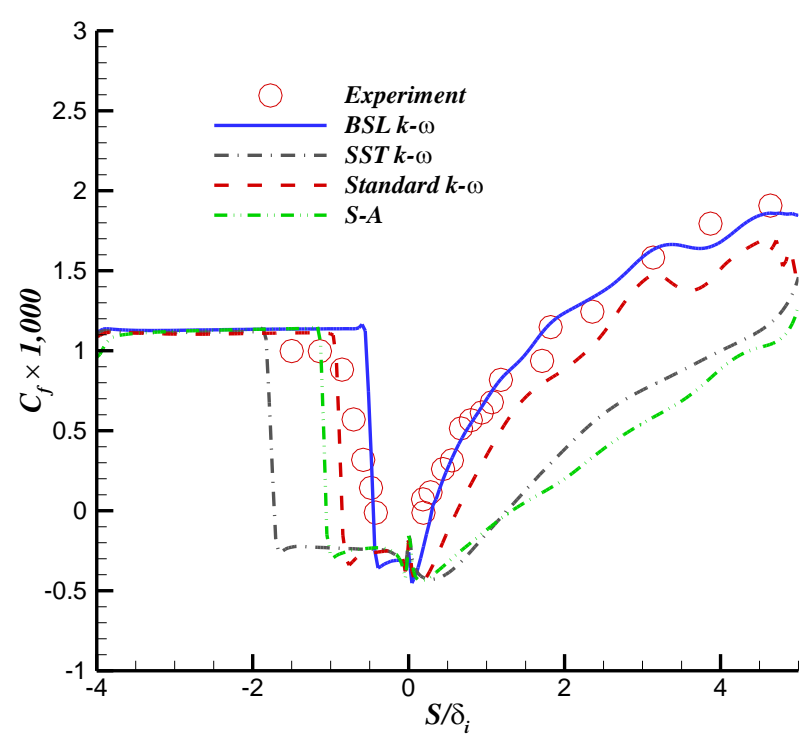

Figure 13. Skin friction coefficient distribution.

Figure 14 and Figure 15 show the prediction results of the remaining turbulence models not previously shown, along with the BSL $\mathrm{k}-\omega$ model with the best prediction performance. The pressure distribution results of the Realizable $\mathrm{k}-\varepsilon$ model indicated by the dotted line reveal that the results are in good agreement with the experimental results in both the separation bubble and the post-reattachment region. However, after the reattachment area, the skin friction coefficient is predicted very high. In the case of the realizable $\mathrm{k}-\varepsilon$ model, since it is a high Reynolds number model that uses a wall function, it is estimated that when there is flow separation, the prediction result is poor. The Stress-BSL model predicts a relatively large separation region due 
to rapid flow separation, but accurately predicts wall pressure and skin friction in the region after reattachment. The stress-omega model predicts the separation region to be too large, and reaches the inlet portion of the computational region. As a result, the convergence problem of CFD analysis occurs, and normal analysis results cannot be obtained.

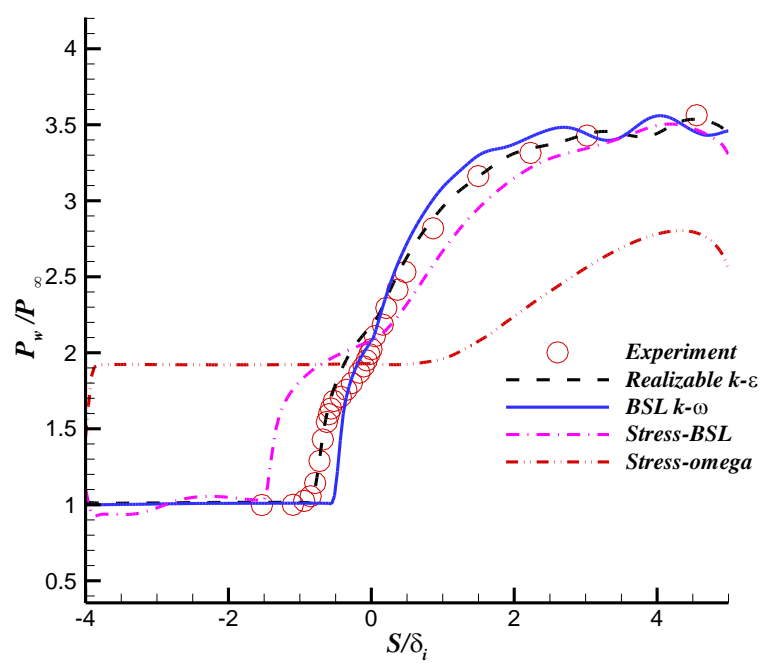

Figure 14. Wall pressure distribution.

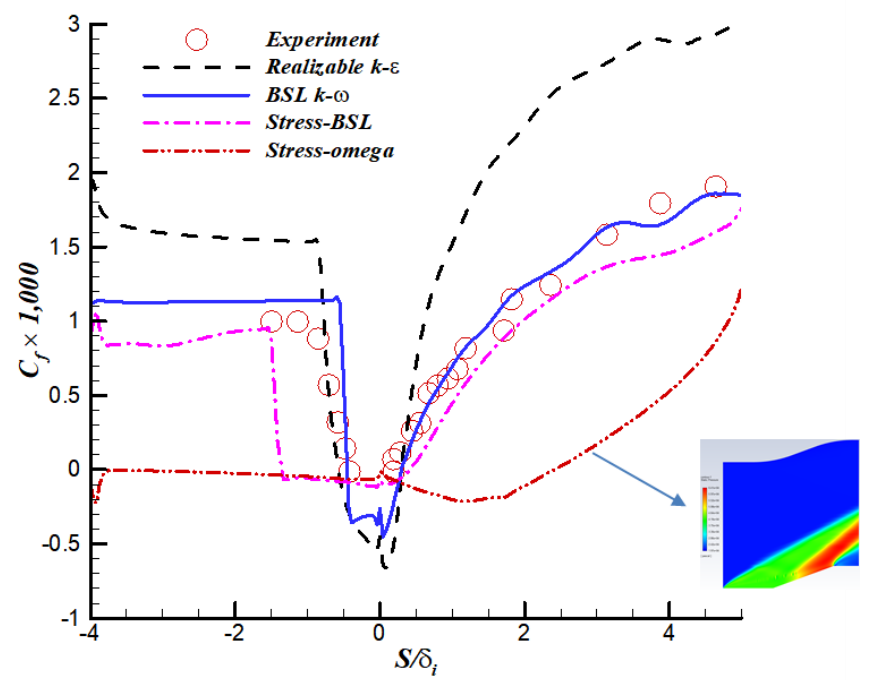

Figure 15. Skin friction coefficient distribution.

\section{Conclusion}

CFD analysis was performed for the $20^{\circ}$ supersonic ramp flow with freestream Mach number 2.79. In order to obtain an accurate solution to the shock wave/Boundary layer interaction problem, adaptive mesh refinement was used; through this, the grid could be concentrated in the region with large gradient of flow variables. The prediction results of 7 turbulence models for SWBLI were compared, and the BSL k- $\omega$ model showed the best prediction results, while the standard k- $\omega$ model also showed good results. The SST k- $\omega$ model, which is one of the most widely used for CFD analysis of various flows, showed problems such as overpredicting the size of the separation region in the supersonic ramp flow covered in this study, and predicting skin friction low in the region after reattachment.

\section{Acknowledgment}

This study was supported by a 2019 research grant from Hanseo University.

\section{References}


1. Settles G. S., Fitzpatrick T. J., \& Bogdonoff S. M. (1979). Detailed study of attached and separated compression corner flowfields in high Reynolds number supersonic flow. AIAA journal, 17(6), 579-585.

2. Settles G. S., \& Dodson L. J. (1994). Supersonic and hypersonic shock/boundary-layer interaction database. AIAA journal, 32(7), 1377-1383.

3. Andreopoulos J., \& Muck K. (1987). Some new aspects of the shock wave boundary layer interaction in compression ramp flows. In 24th Aerospace Sciences Meeting (p. 342).

4. Smits A. J., \& Muck K. C. (1987). Experimental study of three shock wave/turbulent boundary layer interactions. Journal of Fluid Mechanics, 182, 291-314

5. Oliver A., Lillard R., Schwing A., Blaisdell G., \& Lyrintzis A. (2007). Assessment of turbulent shockboundary layer interaction computations using the OVERFLOW code. In 45th AIAA Aerospace Sciences Meeting and Exhibit (p. 104).

6. ANSYS FLUENT 2019R3, Theory \& User guide

7. Spalart, P., \& Allmaras, S. (1992, January). A one-equation turbulence model for aerodynamic flows. In 30th aerospace sciences meeting and exhibit (p. 439).

8. Shih T. H., Liou W. W., Shabbir A., Yang Z., \& Zhu J. (1995). A new k- $\epsilon$ eddy viscosity model for high Reynolds number turbulent flows. Computers \& fluids, 24(3), 227-238.

9. Wilcox, D. C. (2006). Turbulence Modeling for CFD, 3rd edn. La Canada, California: DCW Industries.

10. Menter F. (1993). Zonal two equation k- $\omega$ turbulence models for aerodynamic flows. In 23rd fluid dynamics, plasmadynamics, and lasers conference (p. 2906).

11. Park C. H., \& Park S. O. (2005). On the limiters of two-equation turbulence models. International Journal of Computational Fluid Dynamics, 19(1), 79-86

12. Bardina J. E., Huang P. G., \& Coakley T. J. (1997). Turbulence modeling validation, testing, and development. NASA Technical Memorandum 110446

13. Rizzi A., Jirásek A., Lamar J. E., Crippa S., Badcock K. J., \& Boelens O. J. (2009). Lessons learned from numerical simulations of the F-16XL aircraft at flight conditions. Journal of Aircraft, 46(2), 423-441.

14. Fritz, W. (2007). RANS Solutions for the CAWAPI F-16XL in Solution Adapted Hybrid Grids. 